K. Thanigasalam, Improvement on Davenport's iterative method and new results in additive number theor Wang $Y u a n$, Bounds for solutions of additive equations in an algebraic number field $Y$ 117-144 M. Car, Thëorèmes de densité dans $F_{q}[X]$. . . . . . . . . . . . . . . 145-165

K. Matsumoto, Discrepancy estimates for the value-distribution of the Riemann zeta-

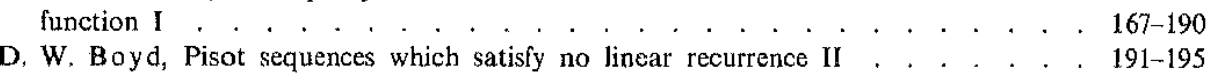
R. F. Tichy, Ein metrischer Satz uber vollständig gleichverteilte Folgen . . . . . 197-207

La revue est consacrée à la Théorie des Nombres

The journal publishes papers on the Theory of Numbers

Die Zeitschrift veröffentlicht Arbeiten aus der Zahlentheorie

Журнал посвящен теории чисел
Liadresse de

la Rédaction

Address of the

Editorial Board

Die Adresse der

Die Adresse der
Schriftleitung und

Алрес редакцин

et de léchange ul. Śniadeckich 8, 00-950 Warszawa

Les auteurs sont priés d'envoyer leurs manuscrits en deux exemplaires The authors are requested to submit papers in two copies

Die Autoren sind gebeten um Zusendung von 2 Exemplaren jeder Arbeit

Рукописи статей редакция просит предлагать в двух экземплярах

(C) Copyright by Państwowe Wydawnictwo Naukowe, Warszawa 1987

ISBN 83-01-07387-X ISSN 0065-1036

PRINTED IN POLAND

\section{Improvement on Davenport's iterative method and new results in additive number theory $\mathrm{III}$}

by

\section{K. Thanigasalam (Monaca, Penn.)}

1. Introduction. In this paper, the results in [9] and [10] (Parts I and II in this series of papers) are improved to the following:

THEOREM 1. Every sufficiently large integer that is $\neq \equiv 0,14$ or $15(\bmod 16)$ is the sum of at most 13 fourth powers.

This improves the corresponding result $G^{*}(4) \leqslant 14$ of Davenport [1].

THEOREM 2. $G(5) \leqslant 21, G(6) \leqslant 32, G(7) \leqslant 45, \quad G(8) \leqslant 62, G(9) \leqslant 82$, $G(10) \leqslant 102, G(11) \leqslant 118$.

Also (in Waring-Goldbach problem), we have (cf. \$2 in Ch. 12 of [6])

THEOREM 3. $H(6) \leqslant 33, H(7) \leqslant 47, H(8) \leqslant 63, H(9) \leqslant 83, H(10) \leqslant 107$; and $h(6) \leqslant 17, h(7) \leqslant 24, h(8) \leqslant 32, h(9) \leqslant 42, h(10) \leqslant 54$.

The author understands that R. C. Vaughan has obtained similar bounds together with his result in [13]. $\left({ }^{1}\right)$ However, differences in the proofs are expected. While the starting point is the same as in [9], further improvement arises from the direct use of Hardy-Littlewood method at the iterative steps. It is acknowledged that for this purpose, Vaughan's result in [12] (see (1.2) below) fits in well for the major arcs. When $3 \leqslant k \leqslant 11$, and $\alpha_{s-1}$ is close to 1 , this is more economical in estimating $\alpha_{s}$ in $U_{s}^{(k)}(N)>N^{\alpha_{s}-z}$. (In essence, we use Weyl's inequality for the $(k-1)$-st power instead of the $k$ th power.) With $g$ denoting the approximating function for $f$ (on major arcs), Hua's result in [5] (which utilises Weil's result in Algebraic Geometry) with a partial summation leads to

$$
f-g \ll q^{(1 / 2)+\varepsilon}\left(1+P^{k}|\beta|\right)
$$

The factor $q^{(1 / 2)+8}$ in (1.1) can be replaced by $q^{(3 / 4)+\varepsilon}$ using the results in [4]. Theorem 2 in [12] is the more precise result

$$
f-g \ll q^{(1 / 2)+\varepsilon}\left(1+P^{k}|\beta|\right)^{1 / 2} .
$$

(1) Copies of [12] and [13] were made available to the author during early January 1985 
In the author's proof, the integration of $F(\alpha)$ over the major arcs is considered rather than that of $f_{s}(\alpha)$ (see (2.1), (2.3) and (2.4)). Nevertheless, for $k=4$, the proof given here requires (1.2) also. Some arguments in [10] (regarding the admissibility of exponents) also play a key role for $k=4$. While (1.1) may be required for $k=5$, for $k \geqslant 6$, it is sufficient to use it with $q^{(3 / 4)+z}$ in place of $q^{(1 / 2)+\varepsilon}$. Also, for $k=5$, one can use Vaughan's result (1.2) replacing $q^{(1 / 2)+\varepsilon}$ with $q^{(3 / 4)+\varepsilon}$, and estimate the integrals of the error terms over the major arcs separately by using (with the standard notations) $\sum_{q} \sum_{a} q^{-4-(2 / 5)-\delta_{0}}|S(a, q)|^{4} \ll 1$. Thus, overall, the proof given here does not

require Weil's result for $k \geqslant 5$ (see $\$ 15 \mathrm{~A}$ ). For uniformity of proofs, we do use (1.2) here for $k=5$. For $k=4,(1.2)$ is used on the major arcs for $f_{4}$ and $f_{5}$ respectively in estimating $\alpha_{5}$ and $\alpha_{6}$.

Some results in [6] for exponential sums with integral-valued polynomials are modified for our use in this paper.

With Davenport's bounds for $\alpha_{r}$ (for suitable $r$ ), generally, we can take $\delta_{i}=1 / 2^{k-2}$ for $i \geqslant r$ in estimating $\alpha_{s}$ with $s \geqslant r+1$ (till $\alpha_{s}$ gets very close to 1 , the impossibility of this choice for $\delta_{i}$ then being seen from the estimates over the major arcs).

2. Some preliminary results. Let (as in [9]) $\varepsilon$ denote an arbitrarily small positive number, and $\delta_{0}$ a small positive constant. All constants implied in the ' $\ll$ ' notation will depend at most on $k$ and $\varepsilon$. Inequalities depending on other parameters (like in Lemmas 2.3 and 2.4) will be so indicated. We recall (from [9]) that with $\lambda_{i}=\left(k-1+\delta_{i}\right) / k, P_{i}=P^{\lambda_{i}}$,

$$
f=\sum_{P<x<2 P} e\left(\alpha x^{k}\right), \quad f_{i}=\sum_{P_{i}<x<2 P_{i}} e\left(\alpha x^{k}\right) .
$$

Let

and (uniformly)

$$
0<\delta \leqslant 1 / 2^{k-2}
$$

$$
\begin{gathered}
F(\alpha)=\sum_{0<1<p^{\delta}} \sum_{P<x<2 P} e\left(\Delta_{t}\left(x^{k}\right) \alpha\right), \\
F_{r}(\alpha)=\sum_{t} \sum_{t_{1}} \ldots \sum_{t_{r}} \sum_{x} e\left(\Delta_{t, t_{1}, \ldots, t_{r}}\left(x^{k}\right) \alpha\right) .
\end{gathered}
$$

With $\left\{\lambda_{1}, \ldots, \lambda_{s}\right\}$ forming admissible exponents, we use Hardy-Littlewood method (with $\delta_{s}=\delta$ ) directly to estimate

$$
S=\int_{0}^{1}|f|^{2}\left|f_{s} f_{s-1} \ldots f_{1}\right|^{2} d \alpha
$$

When Davenport's estimate for $\alpha_{r}$ is used, we replace $\left(f_{1} \ldots f_{r}\right)$ by $U(\alpha)$, where $U(\alpha)=\sum e\left(\alpha u_{i}\right)$, the $u_{i}$ 's being distinct integers of the form $\left(x_{1}^{k}+\ldots\right.$ $\left.+x_{r}^{k}\right)$. The proofs then will have the obvious modifications as in $\$ 9$ in [9]. As explained in [9] (in the proof of the Fundamental Lemma), we have

$$
S \ll P^{1+\varepsilon}\left(P_{s} \ldots P_{1}\right)+I
$$

where (cf. (2.3))

$$
I=\int_{0}^{1} F(\alpha)\left|f_{s} \ldots f_{1}\right|^{2} d \alpha
$$

LeMmA 2.1. If $|\alpha-a / q| \leqslant q^{-2}$, then

$$
F(\alpha) \ll P^{1+\delta+\varepsilon}\left\{P^{-1}+q^{-1}+P^{-k+1-\delta} q\right\}^{1 / 2^{k-2}} \text {. }
$$

Proof. The proof is the same as that of Weyl's inequality (for $(k-1)$-st power). We start with (using Cauchy's inequality)

$$
\begin{aligned}
F(\alpha) & \ll\left(P^{\delta}\right)^{1 / 2}\left\{\sum_{t<p^{\delta}} \sum_{x} \sum_{y} e\left(\left(\Delta_{t}\left(x^{k}\right)-\Delta_{t}\left(y^{k}\right)\right) \alpha\right)\right\}^{1 / 2} \\
& \ll\left(P^{\delta}\right)^{1 / 2}\left\{P^{1+\delta}+\mid F_{1}(\alpha)\right\}^{1 / 2},
\end{aligned}
$$

where $F_{1}(\alpha)$ is defined by (2.3) with $0<t_{1}<P$.

Generally, we have

$$
F_{r}(\alpha) \ll\left(P^{r+\delta}\right)^{1 / 2}\left\{P^{r+1+\delta}+\left|F_{r+1}(\alpha)\right|\right\}^{1 / 2} \quad(1 \leqslant r \leqslant k-3) .
$$

(Indeed, these inequalities are implied in the proof of the Fundamental Lemma in [9].)

At the final step (as in the proof of Weyl's inequality, where the only difference is that we take $\delta=1$ ), by actually carrying out the summation over $x$, and using $t t_{1} \ldots t_{k-2} \ll P^{k-2+\delta}$, we have

$$
F_{k-2}(\alpha) \ll P^{\varepsilon} \sum_{0 \leqslant m \leqslant(k !) P^{k-2+\delta}} \min \left(P,\|\alpha m\|^{-1}\right) .
$$

The sum on the right-side is divided into $\ll\left\{\left(p^{k-2+\delta} / q\right)+1\right\}$ blocks, and we get (in the standard way)

$$
F_{k-2}(\alpha) \ll P^{e}\left\{\left(P^{k-2+\delta} / q\right)+1\right\}\{P+q \log q\} .
$$

Putting these estimates together, we get (2.7).

$$
\text { We have }
$$

$$
\Delta_{t}\left(x^{k}\right)=A_{k-1} x^{k-1}+A_{k-2} x^{k-2}+\ldots+A_{1} x+A_{0}
$$

where

(2.9) $\quad A_{k-1}=k t, \quad A_{k-2}=\left(\begin{array}{l}k \\ 2\end{array}\right) t^{2}, \quad \ldots, \quad A_{1}=k t^{k-1}, \quad A_{0}=t^{k}$, 
so that, $A_{t}\left(x^{k}\right)$ is (for given $t$ ) a polynomial of degree $(k-1)$ in $x$ with positive integral coefficients $A_{k-1}, \ldots, A_{0}$

For every given $t$ with $0<t<P^{\delta}$, let

$$
h_{\mathrm{r}}(\alpha)=\sum_{p<x<2 P} e\left(\Delta_{t}\left(x^{k}\right) \alpha\right)
$$

(so that, from (2.3), $F(\alpha)=\sum_{0<1<p \delta} h_{t}(\alpha)$ );

$$
S_{v}(t)=\sum_{x=1}^{0} e\left(\frac{a}{q}\left(d_{t}\left(x^{k}\right)\right)\right) \quad(1 \leqslant v \leqslant q)
$$

and

$$
S(a, q, t)=S_{q}(t) .
$$

Since $\left(A_{k-1}, \ldots, A_{2}, q\right) \leqslant k t$ and $(a, q)=1$, the next lemma follows from Theorem 2 in $[6]$ (Ch. 1, $\$ 8$ ) (using $t<P^{\delta}$ ).

Lemma 2,2 .

$$
\begin{aligned}
\sum_{1 \leqslant x \leqslant P} e\left(\frac{a}{q}\left(\Delta_{t}\left(x^{k}\right)\right)\right)-(P / q) S(a, q, t) \ll q^{1-1 /(k-1)+\varepsilon} t^{1 /(k-1)} \\
\left.\ll q^{1-1 /(k-1)+\varepsilon} P^{\delta /(k-1)} \text { (uniformly in } t\right) ;
\end{aligned}
$$

and, for $1 \leqslant v \leqslant q$,

$$
\begin{aligned}
S_{v}(t) & \ll q^{1-1 /(k-1)+\varepsilon} t^{1 /(k-1)} \\
& \left.\ll q^{1-1 /(k-1)+\varepsilon} P^{\delta /(k-1)} \quad \text { (uniformly in } t\right) .
\end{aligned}
$$

(Note that the lemma in this form is ineffective if $q<t$. The result then, can be modified. The effect of the factor $t^{1 /(k-1)}$ on the estimates over the major arcs can be reduced or dispensed with by some additional arguments. But, these will not be required for our purposes.)

Lemma 2.3. Let $1 \leqslant l \leqslant k-1$, and with $A_{l}=\left(\begin{array}{c}k \\ k-l\end{array}\right) t^{k-l}$ (cf. (2.9)),

$$
\psi(x)=\psi(x, t)=e\left(\beta A_{l}(q x)^{l}\right) .
$$

Then, if $q \leqslant P^{1-\varepsilon},|\beta| \ll q^{-1} P^{-(l-1)-(k-1) \delta-\varepsilon}$, and $0 \leqslant x \leqslant(2 P / q)$, we have $\left|\psi^{(r)}(x)\right| \leqslant C_{1}(k, r, \varepsilon) P^{-r e} \quad$ (where $C_{1}$ is a suitable constant).

Proof. Let $y=\left(2 \pi \beta A_{i}\right)^{1 / l}(q x)$, so that $\psi(x)=e^{i\left(y^{4}\right)}$. Hence, by Lemma 7.6 in [6],

$$
\left|\psi^{(r)}(x)\right|=\left|\psi(x) G_{r}(y)\left\{\left(2 \pi \beta A_{l}\right)^{1 / l} q\right\}^{r}\right|
$$

where $G_{r}(y)$ is a polynomial of degree $(l-1) r$ in $y$.
Now (from the hypotheses of the lemma),

$\left(|\beta| A_{l}\right)^{1 / l} q \ll q^{1-(1 / l)} P^{-\{(l-1)+(k-l) \delta+\varepsilon ; / l} t^{(k-l) / l}$

$$
\begin{aligned}
& \left.\ll P^{(1-\varepsilon)(1-1 / l)-1+(1-\varepsilon) / l} \quad \text { (using } q \leqslant P^{1-\varepsilon} \text { and } t<P^{\delta}\right) . \\
& \ll P^{-\varepsilon} ;
\end{aligned}
$$

and

$$
\begin{aligned}
\left\{\left(|\beta| A_{i}\right)^{1 / l} q^{l} x^{l-1}\right. & \ll|\beta| A_{l} q^{l}\left(\frac{2 P}{q}\right)^{l-1} \ll q|\beta| t^{k-1} P^{l-1} \\
& \ll P^{-\varepsilon} \quad \text { (using } t<P^{\delta} \text {, and the bound for } q \beta \text { ). }
\end{aligned}
$$

Thus, from (2.15) (with $C_{2}$ denoting a suitable constant), $\left|\psi^{(r)}(x)\right| \leqslant C_{2}(k, r, \varepsilon)\left[1+\left\{\left(|\beta| A_{l}\right)^{1 / l}(q x)\right\}^{(1-1) r}\right]\left\{\left(|\beta| A_{l}\right)^{1 / l} q^{i r} \leqslant C_{1}(k, r, \varepsilon) P^{-r \varepsilon}\right.$.

Lemma 2.4. Let $w_{l}(x)=A_{l} x^{l}+\ldots+A_{0}(1 \leqslant l \leqslant k-1)$ (so that, $w_{k-1}(x)$ $\left.=\Delta_{t}\left(x^{k}\right)\right)$, and $\varphi_{l}(x)=\varphi_{1}(x, t)=e\left(\beta w_{l}(q x)\right)$.

Then, if

(2.16) $q \leqslant P^{1-\varepsilon}, \quad|\beta| \ll q^{-1} P^{-(l-1)-\langle k-1) \delta-\varepsilon} \quad$ and $\quad 0 \leqslant x \leqslant(2 P / q)$,

we have (with a suitable constant $C_{3}$ )

$$
\left|\varphi_{l}^{(r)}(x)\right| \leqslant C_{3}(k, r, \varepsilon) P^{-r x}
$$

Proof. This is proved by induction on $l$. When $l=1$, we have

$$
\varphi_{1}(x)=e\left(\beta\left(A_{1} q x+A_{0}\right)\right)
$$

so that

$$
\left|\varphi_{1}^{(r)}(x)\right|=\left|\left(2 \pi \beta A_{1} q\right)^{r} \varphi_{1}(x)\right| .
$$

Since $A_{1} \ll t^{k-1} \ll P^{(k-1) \delta}$, and $q|\beta| \ll P^{-(k-1) \delta-\varepsilon}$, we see from (2.17) that the result is true for $l=1$.

With $\psi(x)$ as in Lemma 2.3 , we have (for $l \geqslant 2$ )

$$
\varphi_{l}(x)=\psi(x) \varphi_{l-1}(x) .
$$

By inductive assumption for $1-1$, we have

$$
\left|\varphi_{l-1}^{(r)}(x)\right| \leqslant C_{3}(k, r, \varepsilon) P^{-r \varepsilon}
$$

(2.19) $q \leqslant P^{1-\varepsilon}, \quad|\beta| \ll q^{-1} P^{-(l-2)-(k-1+1) \delta-\varepsilon}$ and $0 \leqslant x \leqslant(2 P / q)$.

Since $\delta<1$,

$$
P^{-(l-1)-(k-l) \delta}<P^{-(l-2)-(k-l+1) \delta}
$$


so that, if $\beta$ is subject to (2.16), it also satisfies (2.19). Result now follows from Lemma 2.3 since (from (2.18)),

$$
\varphi_{l}^{(r)}(x)=\psi^{(r)}(x) \varphi_{l-1}(x)+\left(\begin{array}{l}
r \\
1
\end{array}\right) \psi^{(r-1)}(x) \varphi_{l-1}^{\prime}(x)+\ldots+\psi(x) \varphi_{l-1}^{(r)}(x) .
$$

COROLlary 2.4. Let (with $\varphi_{l}(x, t)$ as in Lemma 2.4)

$$
\varphi(x)=\varphi_{k-1}(x, t)=e\left(\beta \Delta_{t}\left((q x)^{k}\right)\right) \text {. }
$$

Then, if

(2.21) $q \leqslant P^{1-\varepsilon}, \quad|\beta| \ll q^{-1} P^{-(k-2)-\delta-\varepsilon}$, and $0 \leqslant x \leqslant(2 P / q)$, we hate

$$
\left|\varphi^{(r)}(x)\right| \leqslant C_{3}(k, r, \varepsilon) P^{-r \varepsilon} .
$$

Proof. In Lemma 2.4, we take $l=k-1$, so that $w_{k-1}(q x)=\Delta_{t}\left((q x)^{k}\right)$. Lemma 2.5. Let $\alpha=(a / q)+\beta$ and

$$
h_{t}^{*}(\alpha)=q^{-1} S(a, q, t) J(\beta) \quad(\text { cf. }(2.12))
$$

where

$$
J(\beta)=\int_{P}^{2 P} e\left(\Delta_{t}\left(x^{k}\right) \beta\right) d x .
$$

The'll. if $q \leqslant P^{1-\varepsilon}$ and $|\beta| \leqslant q^{-1} P^{-(k-2)-\delta-\varepsilon}$, we have (cf. (2,10))

$$
\begin{aligned}
h_{t}(x)-h_{t}^{*}(x) & \ll q^{1-1 /(k-1)+\varepsilon} t^{1 /(k-1)} \\
& \left.\ll q^{1-1 /(k-1)+\varepsilon} p^{\delta /(k-1)} \quad \text { (uniformly in } t\right) .
\end{aligned}
$$

Proof. Starting with Euler's summation formula, this is proved in the same way as Lemma 7.11 in [6] with some modifications. We start with the identity

$$
h_{t}(\alpha)=\sum_{v=1}^{q} e\left(\frac{a}{q} \Delta_{t}\left(v^{k}\right)\right) \Lambda_{v}
$$

where (cf. $(2.20))$

$$
\Lambda_{v}=\sum_{\substack{j \\(P / q)<j+(v / q)<(2 P / q)}} \varphi(j+(v / q)) .
$$

In using Lemma 7.2 in [6], the limits $a, b$ are taken to be $(P / q)$ and $(2 P / q)$ (in place of 0 and $P / q$ ). As in [6], we take $l=[1 / \varepsilon]+1$, so that,

$$
\varphi^{(i)}(x) \ll P^{-1} \quad \text { and } \quad q \int_{P / q}^{2 P / q} \varphi^{(l)}(x) d x \ll 1
$$

Furthermore, we use the inequality (2.14) (in place of the inequality
$S_{v} \ll q^{1-(1 / k)+\varepsilon}$ used in [6]). With these changes, and Corollary 2.4, the arguments are precisely the same as in [6].

Lemma 2.6. With $J(\beta)$ defined as in (2.24),

$$
J(\beta) \ll \min \left(P, P^{2-k} t^{-1}|\beta|^{-1}\right) .
$$

Proof. (For every given $t$ ),

$$
\frac{d}{d x}\left(\Delta_{t}\left(x^{k}\right)\right)=(k-1) A_{k-1} x^{k-2}+\ldots+A_{1},
$$

which is positive, monotonic increasing (for $P<x<2 P$ ), and $\gg t P^{k-2}$ (cf. (2.9)). Hence, the result follows in a standard way by the second Mean-value Theorem. (See for example Lemma 4.2 in [11].)

LeMma 2.7. For every given $t$ (with $h_{t}^{*}(\alpha)$ defined by (2.23) and $\alpha=(a / q)$ $+B)$, we have

$$
h_{t}^{*}(\alpha) \ll q^{-1 /(k-1)+\varepsilon} t^{1 /(k-1)} \min \left(P, P^{2-k} t^{-1}|\beta|^{-1}\right) .
$$

Proof. From (2.14),

$$
S(a, q, t) \ll q^{1-1 /(k-1)+\varepsilon} t^{1 /(k-1)} .
$$

Hence, result follows from Lemma 2.6 .

3. Estimation of $I$. We estimate $I$ (cf. (2.6)) as follows: With $\delta$ satisfying (2.2), let

$$
Q=P^{k-2+\delta+\delta_{0}}
$$

and, divide the unit interval

$$
Q^{-1}<\alpha<1+Q^{-1}
$$

into basic intervals

$$
\text { (3.3) } \quad \mathfrak{M}_{u, q}:|\alpha-a / q| \leqslant(q Q)^{-1} \text { for } 1 \leqslant q<P^{k \delta-\delta_{0}} \text {, }
$$

with $\mathfrak{M}$ denoting their union. Also, let $m$ denote the supplementary intervals in (3.2).

Lemma 3.1. Let $k \geqslant 4$. Then, subject to $(2.2)$, on $m$

$$
\left.F(\alpha) \ll P^{1+\delta_{0}} \quad \text { (cf. }(2.3)\right) \text {. }
$$

Proof. On $m$ with $q \geqslant P^{1-\delta_{0}}$, result follows from Lemma 2.1. Let $x \in m$ with

$$
P^{k \delta-\delta_{0}} \leqslant q \leqslant P^{1-\delta_{0}}
$$

From Lemmas 2.5 and 2.7 (cf. (2.25) and (2.27)), it follows from (3.5) that (for $\left.0<t<P^{\delta}\right)$

$h_{t}(\alpha) \ll P^{1-\delta+\delta_{\rho}}+P^{1-(1 /(k-1))(1-\delta)+\delta_{0}} \ll P^{1-\delta+\delta_{0}}$ (using $\delta \leqslant 1 / 2^{k-2}$ and $\left.k \geqslant 4\right)$. 
Hence,

$$
F(\alpha)=\sum_{t<P^{\delta}} h_{t}(\alpha) \ll P^{1+\delta_{0}},
$$

proving the lemma.

LemMa 3.2. With $\lambda_{1}, \ldots, \lambda_{s}$ and $\alpha_{s}$ defined as before, let

$$
\int_{0}^{1}\left|f_{s} \ldots f_{1}\right|^{2} d \alpha \ll\left(P_{s} \ldots P_{1}\right) P^{\delta_{0}}
$$

Then

$$
\int_{m} F(\alpha)\left|f_{s} \ldots f_{1}\right|^{2} d \alpha \ll P^{1+3 \delta_{0}} P^{k \lambda_{s} \alpha_{s}} .
$$

(Constants multiplying $\delta_{0}$ are not indicated in all the inequalities.)

Proof. The proof follows directly from (3.6) and Lemma 3.1 on noting that

$$
P_{s} \ldots P_{1} \ll P^{k \lambda_{s} \alpha_{s}+\delta_{0}} .
$$

For $k \geqslant 5$, we estimate the integral over $\mathfrak{M}$ as in the next lemma. For $k=4$, some adjustments will be used.

LEMMA 3.3. With the $f_{i}^{\prime}$ 's as occurring in I, suppose that on $\mathfrak{M}$ (for some $r$ with $1 \leqslant r \leqslant k$ )

$$
f_{i} \ll q^{-1 / k} P_{i} \quad \text { for } \quad s-r+1 \leqslant i \leqslant s .
$$

Then

$$
\int_{\mathfrak{M}} F(\alpha)\left|f_{s} \ldots f_{1}\right|^{2} d \alpha \ll P^{\delta /(k-1)} P^{2-k+\varepsilon}\left(P^{2 k \lambda_{s} \alpha_{s}+\delta_{0}}\right) \max \left(1, P^{\mu}\right),
$$

where

$$
\mu=k\{2-(2 r / k)-1 /(k-1)\} \delta .
$$

Proof. From Lemmas 2.5 and 2.7, on $\mathfrak{M}$ (using $t<p^{\delta}$ )

(3.9) $\quad h_{t}(\alpha) \ll q^{-1 /(k-1)+\varepsilon} P^{\delta /(k-1)}\left\{\min \left(P, p^{2-k} t^{-1}|\beta|^{-1}\right)+q\right\}$.

Also, from (3.7),

(3.10)

$$
\left|f_{s} \ldots f_{1}\right|^{2} \ll\left(P_{s} \ldots P_{1}\right)^{2} q^{-(2 r / k)} \ll P^{2 k \lambda_{s} \alpha_{s}+\delta_{0}} q^{-(2 r / k)} .
$$

Hence, from (3.9),

$$
\text { (3.11) } \begin{aligned}
\int_{M i} F(\alpha)\left|f_{s} \ldots f_{1}\right|^{2} d \alpha & =\sum_{0<i<p \delta} \int_{\Re \Re} h_{t}(\alpha)\left|f_{s} \ldots f_{1}\right|^{2} d \alpha \\
\ll\left(P^{2 k \lambda_{s} \alpha_{s}+\delta_{0}}\right) & p^{\delta /(k-1)} \times \\
& \times \sum_{0<t<p^{\delta}}\left\{\sum_{q \leqslant p^{k \delta}-\delta_{0}} \sum_{a} q^{-\left(\frac{1}{k-1}+\frac{2 r}{k}\right)} I(a, q)\right\}
\end{aligned}
$$

(cf. (3.3)), where

Furthermore,

$$
\begin{aligned}
I(a, q) & =\int_{\mathscr{W}_{a, q}}\left\{\min \left(P, P^{2-k} t^{-1}|\beta|^{-1}\right)+q_{j}\right\} d \beta \\
& \ll P^{2-k+\varepsilon} t^{-1}+q(q Q)^{-1} \\
& \ll P^{2-k+\varepsilon} t^{-1}+P^{2-k-\delta-\delta_{0}} \quad \text { (cf. (3.1)). }
\end{aligned}
$$

and

$$
\sum_{0<t<p \delta}\left(P^{2-k+\varepsilon} t^{-1}+P^{2-k-\delta-\delta_{0}}\right) \ll P^{2-k+2 \varepsilon}
$$

$$
\sum_{q \leqslant p^{k \delta}-\delta_{0}} \sum_{a} q^{-\left(\frac{1}{k-1}+\frac{2 r}{k}\right)} \ll \max \left(1, P^{\mu}\right) \quad \text { (cf. (3.8)) }
$$

The lemma now follows from (3.11), (3.12), (3.13) and (3.14).

LemMA 3.4. Let $r$ be chosen with the $f_{i}$ 's satisfying (3.7). Suppose that (with $\alpha_{s}$ and $\delta$ defined as before) either

(a) with $0 \leqslant r \leqslant k-1$,

$$
\text { (b) (with } r \geqslant k)
$$

$$
\text { Then (with } S \text { defined by (2.4)) }
$$

and $U_{s+1}^{(k)}(N)>N^{\alpha_{s+1}-\delta_{0}}$, where

$$
\alpha_{s+1}=(1 / k)+(k-1+\delta) \alpha_{s} / k
$$

Proof. (A) In case (a), from Lemma 3.3 (with $P^{\mu} \gg 1$ ),

$\int_{\mathbb{U}} F(\alpha)\left|f_{s} \ldots f_{1}\right|^{2} d \alpha \ll P^{\delta /(k-1)}\left(P^{(2-k+\varepsilon)}\right) P^{\left(2 k \lambda_{s} \alpha_{s}+\delta_{0}\right)}\left(P^{\mu}\right)$

$$
\ll\left(P^{1+\delta_{0}} P^{k \lambda_{s} \alpha_{s}}\right) P^{\left\{\delta /(k-1)+(1-k+z)+k \lambda_{S} \alpha_{s}+\delta_{0}+\mu\right\}} .
$$

The second factor (with $k \lambda_{s}=k-1+\delta$ ) is

$$
\ll\left\{P^{-(k-1)+2 \delta_{0}}\right\} P^{(k-1+\delta) \alpha_{s}+\mu+\delta /(k-1)},
$$

and, this is easily verified to be $\ll 1$ by using (3.8) and (3.15): Hence, the integral over $\mathfrak{M}$ is $\ll P^{1+\delta_{0}}\left(P^{k \lambda_{s} \alpha_{s}}\right)$.

(B) In case (b), the integral over $\mathfrak{M}$ is

$$
\begin{aligned}
& \ll\left(P^{1+\delta_{0}}\right)\left(P^{k \lambda_{s} \alpha_{s}}\right) P^{-(k-1)+2 \delta_{0}}\left\{P^{(k-1+\delta) \alpha_{s}+\delta /(k-1)}\right\} \\
& <P^{1+\delta_{0}} P^{k \lambda_{s} \alpha_{s}} \quad \text { (from (3.16)). }
\end{aligned}
$$


Thus, from Lemma 3.2 (and (2.4), (2.5), (2.6)), $S \ll P^{1+\delta_{0}} P^{k \lambda_{s} \alpha_{s}}$. Result now follows in a standard way (with $P^{k \lambda_{s} \alpha_{s}} \ll P_{s} \ldots P_{1}$ ).

\section{The case $k=4$.}

Lemma 4.1. Let

$$
\delta_{1}=1, \quad \delta_{2}=3 / 7, \quad \delta_{3}=27 / 103 .
$$

Then, with

$$
\lambda_{3}^{(3)}=\left(3+\delta_{3}\right) / 4, \quad \lambda_{2}^{(3)}=\lambda_{1}^{(3)}=\left(3+\delta_{2}\right) \lambda_{3}^{(3)} / 4
$$

$\left\{\lambda_{1}^{(3)}, \lambda_{2}^{(3)}, \lambda_{3}^{(3)}, 1\right\}$ form admissible exponents, and $U_{4}^{(4)}(N)>N^{\alpha_{4}-\varepsilon}$, where

$$
\alpha_{4}=\left(\lambda_{1}^{(3)}+\lambda_{2}^{(3)}+\lambda_{3}^{(3)}+1\right) / 4=331 / 412
$$

Proof. These are precisely the same bounds obtained by Davenport (for the $\delta$ 's and $\left.\alpha_{3}, \alpha_{4}\right)$ in [1], with the difference that the arguments used there do not establish the admissibility of exponents. For this, we use precisely the same arguments as in the proof of Lemmas 4.2 and 5.1 in [10]. The differences in the details are only computational. (Note that the result $S_{3,6}$ $\ll P_{3} P_{2}^{7 / 2+\varepsilon}$ in Lemma 3.3 of [10] holds for $k=4$ also with $\delta_{2}$ as in (4.1).)

I. Estimation of $\alpha_{5}$. Let

$$
\delta_{4}=0.234
$$

and (with $\delta_{i}(1 \leqslant i \leqslant 3)$ as in $\left.(4.1)\right)$,

(4.4) $\quad \lambda_{4}=\lambda_{4}^{(4)}=\left(3+\delta_{4}\right) / 4, \quad \lambda_{i}=\lambda_{i}^{(4)}=\left(3+\delta_{i}\right) \lambda_{i+1}^{(4)} / 4 \quad(1 \leqslant i \leqslant 3)$,

and define $f, f_{i}(1 \leqslant i \leqslant 4)$ as before.

With $\delta=\delta_{4}$, let the $\mathfrak{M}_{a, q}$ 's be defined as in (3.3) with

$$
1 \leqslant q<P^{4 \delta_{4}-\delta_{0}} \text {. }
$$

LeMMA 4.2. On $\mathfrak{M}$
(a) $f_{i} \ll q^{-v_{2}} P_{2}(i=1,2)$ (cf. $\left.f_{1}=f_{2}\right)$,
(b) $f_{3} \ll q^{-v_{3}} p_{3}$,
(c) $f_{4} \ll q^{-v_{4}} P_{4}$,

where

(4.6) $\quad v_{2}=0.103805, \quad v_{3}=0.204442, \quad v_{4}=1 / 4$.

Proof. From (1.2) (with $g_{i}$ denoting the approximating function to $f_{i}$ ),

$$
f_{i} \ll g_{i}+q^{1 / 2+\varepsilon}\left(1+P_{i}^{4}|\beta|\right)^{1 / 2} .
$$

With the estimate $g_{i} \ll q^{-1 / 4} P_{i}$, it is easily verified that

$$
f_{i} \ll q^{-1 / 4} P_{i}+q^{1 / 2+\varepsilon} \quad(1 \leqslant i \leqslant 4) .
$$

Now, by computing $\lambda_{i}(1 \leqslant i \leqslant 4)$, and using (4.5), (a), (b), and (c) are verifed from (4.8).
Lemma 4.3. (With $S$ as in (2.4).) $S \leqslant P^{1+\delta} P^{\left(4 \lambda_{4} \alpha_{4}+\delta_{0}\right)}$ $U_{5}^{(4)}(N)>N^{\alpha_{5}-\dot{\delta}_{0}}$, where

$$
\alpha_{5}=(1 / 4)+\lambda_{4}^{(4)} \alpha_{4}>0.8995472
$$

Proof. We estimate $S$ (cf. (2.4)) as in the general case. Since $\delta_{4}<1 / 4$, the integral over $m$ (arguing as in the general case) is
(4.10).
$\ll P^{1+\delta_{0}} P^{\left(4 \lambda_{4} \alpha_{4}+\delta_{0}\right)}$
(cf. Lemmas 3.1 and 3.2).

For the estimate of the integral over $\mathfrak{M}$, we use Lemma 3.3 replacing $\mu$ by $\mu^{\prime}$, where

$$
\mu^{\prime}=4(2-2 v-1 / 3) \delta_{4}
$$

with

$$
v=2 v_{2}+v_{3}+v_{4}=0.662052 .
$$

It is now verified from (4.2), (4.3), (4.4), (4.6), (4.11) and (4.12) (and Lemma 3.3) that the integral over $\mathfrak{M}$ is also bounded by the estimate in (4.10). Result now follows with $\alpha_{5}$ as in (4.9).

II. Estimation of $\alpha_{6}$. Let

$$
\delta_{5}=0.1985
$$

and (with $\delta_{i}(1 \leqslant i \leqslant 4)$ as above),

$$
\lambda_{5}=\lambda_{5}^{(5)}=\left(3+\delta_{5}\right) / 4, \quad \lambda_{i}=\lambda_{i}^{(5)}=\left(3+\delta_{i}\right) \lambda_{i+1}^{(5)} / 4 \quad(1 \leqslant i \leqslant 4) .
$$

Let the $\mathfrak{M}_{a, q}$ 's be defined with

$$
1 \leqslant q<P^{4 \delta_{5}-\delta_{0}}
$$

\section{Lemma 4.4. On $\mathfrak{M}$}

$$
\text { (a) } f_{i} \ll q^{-v_{2}^{\prime}} P_{2}(i=1,2), \quad \text { (b) } f_{3} \ll q^{-v_{3}^{\prime}} P_{3}
$$$$
\text { (c) } f_{4} \ll q^{-v_{4}^{\prime}} P_{4}, \quad \text { (d) } f_{5} \ll q^{-v_{5}^{\prime}} P_{5} \text {, }
$$

where

$$
v_{2}^{\prime}=0.069167, \quad v_{3}^{\prime}=0.164025, \quad v_{4}^{\prime}=v_{s}^{\prime}=1 / 4 .
$$

Proof. This is verified from (4.8), (4.14) and (4.15) by computing $\lambda_{i}$ $(1 \leqslant i \leqslant 5)$.

\section{Lemma 4.5 .}

$$
S=\int_{0}^{1}|f|^{2}\left|f_{5} \ldots f_{1}\right|^{2} d \alpha \ll P^{1+\delta_{0}} P^{\left(4 \lambda_{5} \alpha_{5}+\delta_{0}\right)}
$$

and:

$$
U_{6}^{(4)}(N)>N^{\alpha_{6}-\delta_{0}},
$$




$$
\alpha_{6}=(1 / 4)+\lambda_{5}^{(5)} \alpha_{5}>1-(1 / 32)+\left(5 / 10^{4}\right) .
$$

Proof. Same as Lemma 4.3, where in place of (4.11) and (4.12), we use

$$
\mu^{\prime}=4\left(2-2 v^{\prime}-1 / 3\right) \delta_{5}^{\prime} \quad \text { with } \quad v^{\prime}=2 v_{2}^{\prime}+v_{3}^{\prime}+v_{4}^{\prime}+v_{5}^{\prime}=0.802359
$$

Proof of Theorem 1. We follow the same proof as in $\$ 11$ of [10] by considering $\mathfrak{M}_{a, q}$ 's with $|\alpha-a / q| \leqslant q^{-1} P^{-3-\delta_{0}}$ for $1 \leqslant q \leqslant P^{1 / 2}$. For the treatment of the minor arcs $m$, we use (4.17) and. Weyl's inequality for an additional 4-th power. Note that with admissibility of exponents, the treatment of the singular series is simplified considerably (as there is no need to impose congruence conditions on the summands in defining the exponential sums). It now follows in a standard way that

$$
G^{*}(4) \leqslant 2(6)+1=13 \text {. }
$$

5. The case $k=5$. We start with Lemma 7.1 in $[10]$ taking $\delta_{i}(1 \leqslant i \leqslant 5)$ as in (3.3) (in [10]). Then $\left\{\lambda_{1}^{(5)}, \ldots, \lambda_{5}^{(5)}, 1\right\}$ form admissible exponents, and (cf. (7.3) in [10])

$$
0.823065<\alpha_{6}<0.823069 .
$$

In the estimates of $\alpha_{7}, \alpha_{8}$ and $\alpha_{9}$ (corresponding to (4.8)), we now use

$$
f_{i} \ll q^{-1 / 5} P_{i}+q^{1 / 2+\varepsilon}
$$

We can successively take $\delta_{i}=1 / 8$ for $6 \leqslant i \leqslant 8$.

In estimating $\alpha_{7}, \alpha_{8}$ and $\alpha_{9}$, we use $f_{i} \ll q^{-1 / 5} P_{\mathrm{i}}$ (as easily verified from (5.2)) respectively with $3 \leqslant i \leqslant 6 ; 4 \leqslant i \leqslant 7$; and $5 \leqslant i \leqslant 8$. (3.15) is satisfied with $k=5, \delta=1 / 8$ and $r=4$ in each of these cases. (Actually, it is sufficient to take $r=3$ in estimating $\left.\alpha_{7}\right)\left(\alpha_{10}\right.$ may also be estimated with $\delta_{9}$. slightly less than $1 / 8$.)

Thus, starting with (5.1), and using

$$
\alpha_{i+1}=1 / 5+\alpha_{i}\{4+(1 / 8)\} / 5=1 / 5+(33 / 40) \alpha_{i} \quad(6 \leqslant i \leqslant 8),
$$

we have

Lemma 5.1 .

$$
S=\int_{0}^{1}|f|^{2}\left|f_{8} \ldots f_{1}\right|^{2} d \alpha \ll P^{1+\delta_{0}}\left(P_{8} \ldots P_{1}\right)
$$

and

$$
\alpha_{9}>0.963288>1-(3 / 80)+\left(7 / 10^{4}\right) .
$$

Proof that $G(5) \leqslant 21$. We follow precisely the same proof in $\S 11$ of [10] introducing three more 5-th powers to deal with $m$. With Weyl's inequality, the saving contributed on $m$ by these three 5-th powers is $N^{-(3 / 80)+\delta_{0}}$.
Hence, from (5.4), it follows that

$$
G(5) \leqslant 2(9)+3=21 .
$$

6. The case $k=6$. The next two lemmas will be used for $k \geqslant 6$. LeMma 6.1. Let

$$
\lambda=\left\{(k-1)+\left(1 / 2^{k-2}\right)\right\} / k .
$$

Then, for $k \geqslant 6$,

$$
\lambda^{k}>k\left\{(3 / 4+(1 / k)\} / 2^{k+2} .\right.
$$

Pro of. For $k=6$, this can be verified numerically. Also, $\lambda^{k}>(1-1 / k)^{k}$ 。 and $(1-1 / k)^{k}$ is an increasing function of $k$, while the right-side of $(6.2)$ is a decreasing function. Hence, result follows with verification for the case $k=7$.

LEMmA 6.2. Let $1 \leqslant q \leqslant P^{\left(k / 2^{k-2}\right)}$. Then, with $\hat{\lambda}$ 'as in (6.1), we have

$$
P^{(2 r)}>q^{3 / 4+(1 / k)+\varepsilon} \text { for } \quad 1 \leqslant r \leqslant k .
$$

Proof. The proof follows directly from (6.3).

Nore. If (1.2) is used, we can replace $1 / 2$ by $3 / 4$ in (6.3). Corresponding to $(5.2)$, we use $f_{i} \ll q^{-1 / k} P_{i}+q^{(3 / 4)+\varepsilon}$, which, with (6.2) gives (on major arcs)

$$
f_{i} \ll q^{-1 / k} P_{i}
$$

for all the $f_{i}$ 's considered in the proofs.

Let $U(\alpha)=\sum_{u_{i}} e\left(\alpha u_{i}\right)$, where the $u_{i}$ 's are distinct integers of the form $\left(\sum_{i=1}^{7} x_{i}^{6}\right)$. occurring in the estimate of $\alpha_{7}$ in [6]. The next lemma follows from [6] (Ch. 9, Lemma 9.9).

Lemima 6.3 .

$$
\int_{0}^{1}|f|^{2}|U(\alpha)|^{2} d \alpha \ll P^{1+\varepsilon} U(0)
$$

and

where

$$
U_{8}^{(6)}(N)>N^{\alpha}
$$

$$
\alpha_{8}=0.8283548
$$

Lemma 6.4. With $f, f_{i}$ s and $U(\alpha)$ defined (iteratively) as before,

$$
S=\int_{0}^{1}|f|^{2}\left|f_{14} f_{13} \ldots f_{8}\right|^{2} \cdot|U(\alpha)|^{2} d \alpha \ll P^{1+\delta_{0}}\left(P_{14} \ldots P_{8}\right) U(0),
$$


and

where

$$
U_{15}^{(6)}(N)>N^{\alpha_{15}}
$$

$$
\alpha_{15}>0.9941155>1-(2 / 192)+\left(4 / 10^{3}\right) .
$$

Proof. We take $\delta_{i}=1 / 16$ for $8 \leqslant i \leqslant 14$ (and use (6.4) with $k=6$ ). At the first step (of estimating $\alpha_{9}$ ), we take $\delta=1 / 16$ and $r=1$ in (3.15). (Actually, it is sufficient to take $r=0$, and use trivial estimate for $f_{8}$ over the major arcs.) Then $r$ is taken one larger at each of the successive steps (of estimating $\alpha_{10}, \alpha_{11}, \ldots$ ). When $r=6$, we use (3.16). The rest of the proofs are simple computational verifications (with (3.15) and (3.16)). Using

(6.9) $\quad \alpha_{i+1}=1 / 6+\alpha_{i}\{5+(1 / 16)\} / 6=1 / 6+(27 / 32) \alpha_{i} \quad(8 \leqslant i \leqslant 14)$,

$\alpha_{15}$ is computed to satisfy (6.8).

Proof that $G(6) \leqslant 32$. We introduce two additional 6-th powers (for dealing with the minor arcs $m$ ) and use (6.8). We follow the proof of Lemma 4.8 in [9] defining $\mathfrak{M}_{a, q}$ 's with $1 \leqslant q \leqslant P^{3 / 16}$. The saving contributed by the two additional 6-th powers over $m$ (using Weyl's inequality) is $N^{-(2 / 192)+\delta_{0}}$. The result

$$
G(6) \leqslant 2(15)+2=32
$$

now follows in a standard way from (6.8).

7. The case $k=7$. For $k \geqslant 7$, conditions (3.15) and (3.16) are verified as in the case $k=6$ (with enough to spare in the estimates) with $\delta=1 / 2^{k-2}$. So, we indicate only the main results in these cases.

With $U(\alpha)=\sum_{u_{i}} e\left(\alpha u_{i}\right)$, where the $u_{i}$ s are distinct integers of the form $\left(\sum_{i=1}^{10} x_{i}^{7}\right)$ occurring in the estimate of $\alpha_{10}$ (cf. Lemma 9.10 in [6]), and starting with

$$
\alpha_{11}=0.85627
$$

we compute the $\alpha$ 's with

(7.2) $\alpha_{i+1}=1 / 7+\alpha_{i}\{6+(1 / 32)\} / 7=1 / 7+(193 / 224) \alpha_{i} \quad(11 \leqslant i \leqslant 21)$.

We have

LEMMA 7.1 .

(7.3) $\quad S=\int_{0}^{1}|f|^{2}\left|f_{21} \ldots f_{11}\right|^{2}|U(\alpha)|^{2} d \alpha \ll P^{1+\delta_{0}}\left(P_{21} \ldots P_{11}\right) U(0)$,

and

$$
U_{22}^{(7)}(N)>N^{\alpha 2}
$$

where

$$
\alpha_{22}>0.998068>1-(1 / 448)+\left(2 / 10^{4}\right) .
$$

We now introduce an additional 7-th power, and follow the proof of Lemma 4.8 in [9]. It is verified from (7.4) that there is sufficient saving over $m$ (with Weyl's inequality for the additional 7-th power); so that

$$
G(7) \leqslant 2(22)+1=45 \text {. }
$$

8. The case $k=8$. Let $U(\alpha)=\sum_{u_{i}} e\left(\alpha u_{i}\right)$, where the $u_{i}$ 's are distinct integers of the form $\left(\sum_{i=1}^{13} x_{i}^{8}\right)$ occurring in the estimate of $\alpha_{13}$ (cf. Lemma 9.11 in [6]). Starting with

$$
\alpha_{14}=0.8714775
$$

we compute the $\alpha$ 's with

(8.2) $\quad \alpha_{i+1}=1 / 8+\alpha_{i}\{7+(1 / 64)\} / 8=1 / 8+(449 / 512) \alpha_{i} \quad(14 \leqslant i \leqslant 29)$

to get the following:

LEMMA 8.1.

(8.3) $\quad S=\int_{0}^{1}|f|^{2}\left|f_{29} \ldots f_{14}\right|^{2}|U(\alpha)|^{2} d \alpha \ll P^{1+\delta_{0}}\left(P_{29} \ldots P_{14}\right) U(0)$

and

$$
U_{30}^{(8)}(N)>N^{\alpha} 30
$$

where

$$
\alpha_{30}>0.998205>1-(2 / 1024)+\left(1 / 10^{4}\right) .
$$

Now, with (Weyl's inequality for) two additional 8-th powers, we get from (8.4)

$$
G(8) \leqslant 2(30)+2=62
$$

9. The case $k=9$. Let $u_{i}$ 's denote distinct integers of the form $\left(\sum_{i=1}^{16} x_{i}^{9}\right)$ occurring in the estimate of $\alpha_{16}$. With the estimates as in $\$ 7$ of [7], we can take

$$
\alpha_{17}=0.882015
$$

Now, the $\alpha$ 's are computed with

$(9.2)$

$$
\alpha_{i+1}=(1 / 9)+\alpha_{i}\{8+(1 / 128)\} / 9=(1 / 9)+(1025 / 1152) \alpha_{i} . \quad(17 \leqslant i \leqslant 39) .
$$


We have

LEMMA 9.1.

(9.3) $\quad S=\int_{0}^{1}|f|^{2}\left|f_{39} \ldots f_{17}\right|^{2}|U(\alpha)|^{2} d \alpha \ll P^{1+\delta_{0}}\left(P_{39} \ldots P_{17}\right) U(0)$

and

$$
U_{40}^{(9)}(N)>N^{\alpha} 40
$$

where

$$
\alpha_{40}>0.9993>1-(2 / 2304)+\left(1 / 10^{4}\right)
$$

Now, with (Weyl's inequality for) two additional 9-th powers, we get (from (9.4))

$$
G(9) \leqslant 2(40)+2=82 .
$$

10. The cases $k=10$ and 11 .

(A) $k=10$. With the estimates as in (b) of $\$ 7$ in [7], we have

$$
U_{20}^{(10)}(N)>N^{\alpha 20}
$$

where

$$
\alpha_{20}=0.89095
$$

Now, we compute the $\alpha$ 's with

$$
\alpha_{i+1}=(1 / 10)+\alpha_{i}\{9+(1 / 256)\} / 10=(1 / 10)+(461 / 512) \alpha_{i},
$$

to get

(10.3)

$$
\alpha_{30}>0.9643594
$$

$$
\alpha_{36}>0.9828417 \text {, }
$$

and (as required in the estimate of $H(10)$ )

$$
\alpha_{51}>0.999553>1-(3 / 5120)+\left(1 / 10^{4}\right) .
$$

Condition (14) in [8] is satisfied with $s_{1}=30, s_{2}=36, \gamma_{1}=\alpha_{30}$, and $\gamma_{2}$ $=\alpha_{36}^{\prime}$, so that

$$
\text { (10.6) }
$$$$
G(10) \leqslant 2(36)+30=102 .
$$

(B) $k=11$. As in (c) of $\$ 7$ in [7], we have $U_{24}^{(11)}(N)>N^{\alpha 24}$, where

$$
\alpha_{24}=0.90774 \text {. }
$$

The $\alpha$ 's are now computed with

$(10.8) \quad \alpha_{i+1}=(1 / 11)+\alpha_{i}\{10+(1 / 512)\} / 11=1 / 11+(5121 / 5632) \alpha_{i}$, giving

$$
\alpha_{34}>0.96556 \text { and } \alpha_{42}>0.98495 \text {. }
$$

Condition (14) in [8] is satisfied with $s_{1}=34, s_{2}=42, \gamma_{1}=\alpha_{34}$ and $\gamma_{2}=\alpha_{42}$. Hence,

$$
G(11) \leqslant 2(42)+34=118 .
$$

11. Proof of Theorem 3. For $6 \leqslant k \leqslant 9$, Theorem 3 follows from Theorem 2 as indicated in $\$ 17$ of [9]. For $k=10$, using (10.5) and Weyl's inequality for 3 additional 10-th powers (in place of the method in [8]), we get $G(10) \leqslant 2(51)+3=105$, and this implies that $H(10) \leqslant 107$.

12. A theorem on admissible exponents. The next theorem is a slight improvement for $4 \leqslant k \leqslant 8$ on the corresponding result of Davenport and Erdös in [3]: $\alpha_{3}$ is computed with $\alpha=\alpha_{2}=2 / k$, and $l=2$ in Theorem 2 of [2]. But, the additional arguments as in Lemma 2.5 of [10] are required to establish the admissibility of exponents.

THEOREM 4. Let $4 \leqslant k \leqslant 8$ and $\lambda=3 k /(3 k+2)$. Then $\{1, \lambda, \lambda\}$ form admissible exponents.

13. Precise bounds for $U_{s}^{(5)}(N)$ and $U_{s}^{(6)}(N)$. The bounds for $U_{s}^{(5)}(N)$ and $U_{s}^{(6)}(N)$ so far used in the proofs work in the estimates of both $G(k)$ and $H(k)$. However, the bounds can be made more precise by starting with Davenport's estimates for the $\alpha_{i}$ 's (with small $i$ 's). This is made possible only because the major arcs are considered with small values of $q$, namely $q \leqslant P^{k / 2^{k-2}}$. With the $\alpha$ 's defined as before, we have the following:

THEOREM 5. (a) For $k=5$,

(13.1) $\quad \alpha_{4}=569 / 845, \quad \alpha_{5}=\left(7+33 \alpha_{4}\right) / 5\left(7+\alpha_{4}\right)=6173 / 8105$,

$$
\alpha_{s}=\left(8+33 \alpha_{s-1}\right) / 40 \quad(6 \leqslant s \leqslant 9) \text {. }
$$

In particular,

$$
\alpha_{8}=\frac{481782661}{518720000} \quad \text { and } \quad \alpha_{9}=\frac{20048587813}{20748800000} .
$$

(b) For $k=6$,

$$
\alpha_{6}=575117 / 787182, \quad \alpha_{s}=\left(15+81 \alpha_{s-1}\right) / 6\left(15+\alpha_{s-1}\right)
$$

$$
\alpha_{s}=\left(16+81 \alpha_{s-1}\right) / 96 \quad(9 \leqslant s \leqslant 15)
$$

$(7 \leqslant s \leqslant 8)$

Here,

$$
\alpha_{8}=\frac{649358333}{781898958}
$$


Proof. Of these, (13.1) and (13.4) are Davenport's and are given in [2]. With the arguments already given in the paper, for $k=5$, we take $\delta_{i}=1 / 8$ for $5 \leqslant i \leqslant 8$, and use $\alpha_{s}=1 / 5+(33 / 40) \alpha_{s-1}$ leading to (13.2); for $k=6$, we take $\delta_{i}=1 / 16$ for $8 \leqslant i \leqslant 14$, and use $\alpha_{s}=1 / 6+(27 / 32) \alpha_{s-1}$, which establishes (13.5).

It should be noted here that in Davenport's results, $x_{5}$ can be kept explicit for $k=5$, and $x_{8}$ for $k=6$ (in estimating the number of integers of the forms $x_{1}^{5}+\ldots+x_{5}^{5}$, and $\left.x_{1}^{6}+\ldots+x_{8}^{6}\right)$. For $k=5$, it is also necessary to modify the arguments (for the major arcs) as indicated in the introduction. In this connection, Vaughan's result (1.2) may have to be used (where, it would be sufficient to work with $q^{(3 / 4)+i}$ in place of $q^{(1 / 2)+\varepsilon}$ ). Also, the integrals (over the major arcs) of the error terms are to be estimated separately.

14. Addendum. For $k=6$, Davenport's estimate of $\alpha_{8}$ allows only $x_{8}$ to be explicit in $x_{1}^{6}+\ldots+x_{8}^{6}$. The methods in [9] and [10] (as in the case $k$ $=5)$ allow the choice of the parameters $\delta_{i}(1 \leqslant i \leqslant 7)$ close to that of Davenport's, at the same time retaining the use of admissible exponents (so that, $x_{1}, \ldots, x_{8}$ are all explicit). These were used in the author's earlier estimate $G(6) \leqslant 34$.

\section{Additional remarks.}

(A) In the proof of $G(k) \leqslant k\{3 \log k+\log 108\}$ (in [7]), the author used (1.1) for the treatment of major arcs, and the same proofs were utilised in [8] also. However, it is now seen that it would be sufficient to use (1.1) with $q^{(3 / 4)+\varepsilon}$ replacing $q^{(1 / 2)+\varepsilon}$. For this (with the notations as in [7], with the major arcs defined as in [8], and with $h=[(k+1) / 2])$, the integrals of $E_{i}$ $(0 \leqslant i \leqslant k)$ where $E_{0}=\left(f^{2}-g^{2}\right)\left(f_{1} \ldots f_{k}\right)^{2}$, and for $1 \leqslant i \leqslant k, \quad E_{i}$ $=g^{2}\left(g_{1} \ldots g_{i-1}\right)^{2}\left(f_{i}^{2}-g_{i}^{2}\right)\left(f_{i+1} \ldots f_{k}\right)^{2}$ are estimated separately over the major arcs using

$$
\sum_{q} \sum_{a} q^{-(2 i+1)+(2 i-3) / k}|S(a, q)|^{2 i} \ll 1 \quad \text { for } \quad 2 \leqslant i \leqslant h
$$

and also

$$
\sum_{q} \sum_{a} q^{-\left(2 h+2+\delta_{0}\right)}|S(a, q)|^{2 h+.2} \ll 1 .
$$

Here, the results of Davenport and Heilbronn in [4] provide sufficiently good estimates over the major arcs for $\left(f_{i}-g_{i}\right)$ with $1 \leqslant i \leqslant h$. For $h+1 \leqslant i \leqslant k$, a non-trivial estimate for $\left(f_{i}-y_{i}\right)$ would suffice. This may be obtained by using Weyl's inequality for $f_{i}$ over the major arcs with large $q$ (and standard estimate for $g_{i}$ ).

(B) For $k=5$, the recurrence relation (13.2) may be established with $s$ $=9$ also either by (i) removing the effect of the factor $t^{(1 / k-1)}$ (arising from (2.14) by using a suitable summation technique over the major arcs; or (ii) by considering the integral of $f_{9}(x)$ rather than that of $F(\alpha)$ over the major arcs.
(C) For $k=4$, more precise bounds for $\alpha_{5}$ and $\alpha_{6}$ can be obtained as follows: As mentioned in $(\mathrm{B})$, remove the effect of the factor $t^{1 /(k-1)}$, and integrate the error terms over the major arcs separately using the inequality

$$
\sum_{q} \sum_{a} q^{-\left(4+1 / 4+\delta_{0}\right)}|S(a, q)|^{4} \ll 1
$$

In addition to these, in estimating $\alpha_{6}$, the major arcs may be split into two sets $\mathfrak{M}_{1}$ and $\mathfrak{M}_{2}$ (those with small and large $q$ 's respectively). $\mathfrak{M}_{1}$ is treated as in the paper. In treating $\mathfrak{M}_{2}, f_{2}$ is better estimated by using Weyl's inequality, rather than using the estimates for major arcs.

(D) The reference no [17] given in [9] to the author's paper is changed to Portugaliae Math. 42 (4) (1985), pp. 447-465.

Added in proofs: (1) The case $k=6$. The author has withheld a (previously planned) separate publication for the case $k=6$ as further improvements have been obtained since the announcement of the method, the latest being $G(6) \leqslant 31$. The bound for $\alpha_{15}$ given by (13.5) falls only slightly short for proving $G(6) \leqslant 2(15)+1=31$. This gap can be filled. One way of filling this gap is to iterate with Hardy-Littlewood method at the last step also in dealing with the minor ares (without separately using Weyl's inequality for a 6 -th power) to get $G(6)$ $\leqslant 15+16=31$. Here, we do not estimate $x_{16}$ as we already know that $z_{16}=1$ (and that it cannot exceed 1), but obtain a better saving than what is provided by Weyl's inequality. With $\mu$ corresponding to $\alpha_{16}$ given by $(13.5)$, and $\beta=\left(\alpha_{15}+\mu\right) / 2$, the saving on the minor arcs (by a combination with Schwarz's inequality) would be $N^{-\beta} \ll N^{-1-\delta_{0}}$. In fact it would be sufficient to work with a number slightly less than $\mu$, so that the major arcs need be considered with even smaller values of $q$ (than the ones already considered with $q<p^{3 / 8}$ ). This would further simpify the treatment of major arcs. While putting these together requires an additional argument, a closer reflection will reveal that the crucial idea is the iterative use of Hardy-Littlewood method with $F(\alpha)$ (cf. (2.3)). This is explicitly and clearly stated in Part I ([9]). What makes the iteration successful is the fact that $t$ is taken in a small interval (as compared to $x$ ). (2). In the footnote of page 1 of Part II ([10]), the equality $\delta_{i}=(1 / 8)$ bas appeared incorrectly with 118 in place of $(1 / 8)$.

\section{References}

[1] H. Daven port, On Waring's prohlem for fourth powers, Ann. Malh. 40 (1939), pp. 731 747 .

[2] - On sums of positive integral $k$-th powers, Amer. J. Math. 64 (1942), pp. 189-198.

[3] H. Davenport and P. Erdös, On sums of positice integral k-th powers, Ann. Mally. 40 (1939), pp. 533-536.

[4] H. Davenport and H. Heilbronn, On an exponential sum, Proc. London Math. Soc. (2) 41 (1936), pp. 449-453

[5] L. K. Hua, On exponential sums, Science Record (N. S) 1 (1) (1957), pp. 1-4.

[6] - Additive Primzahltheorie, Leipzig 1959.

[7] K. Thanigasalam, On Wuring's problem, Acta Arith. 38 (1980), pp. 141-155.

[8] - Some new estimates for $G(k)$ in Waring's mroblem, ibid. 42 (1982), pp. 73-78.

[9] - Improvement on Davenport's iterative method and new results in additive number theory I, ibid. $46(1985)$, pp. 1-31.

[10] -, - II, ibid. 46 (1986), pp. 91-112. 
[11] E. C. Titchmatsh, The theory of the Riemann Zeta-Function, Clarendon Press, Oxford 1951.

[12]. R. C. Vaughan, Some remarks on Weyl sums, Topics in Classical Number Theory, Colioquia Math. Soc. János Bolyai 34, Budapest 1981 (Elsevier, North-Holland, 1984), pp. $1585-1602$

[13] - Sums of three cubes, Bult. London Math. Soc. 17 (1985), pp. 17-20.

DEPARTMENT OF MATHEMATICS PENNSYLVANIA STATE UNIVERSITY Baitye: Cumpuls, Monacial, PA 15061

Editor's note. The results of Vaughan referred to in the introduction have already appeared in print, see R. C. Vaughan, On Waring's problem for smaller exponents, Proc. London Math Soc. (3) 52 (1986), pp. 445-463, and On Waring's problem for sixth powers, J. London Math. Soc. (2) 33 (1986), pp, 227-236.

\section{Bounds for solutions of additive equations in an algebraic number field $I$}

by

\author{
WANG YUAN* (Beijing, China)
}

1. Introduction. Let $k$ be a rational integer $\geqslant 1$. Similar to Waring's problem, one can show by the Hardy-Littlewood's method that an equation

$$
a_{1} x_{1}^{k}+\ldots+a_{s} x_{s}^{k}=0
$$

where $a_{1}, \ldots, a_{s}$ are given rational integers but not all of the same sign, has a nontrivial solution in nonnegative rational integers $x_{1}, \ldots, x_{s}$, provided only that $s \geqslant c_{1}(k)$. (See, e.g., H. Davenport [3]). Here we use $c(f, \ldots, g)$ to denote a positive constant depending on $f, \ldots, g$. As for a bound of these solutions, it was shown by J. Pitman [10] that if $s \geqslant c_{2}(k)$, then there exists a nontrivial solution in nonnegative integers such that

$$
\max _{i} x_{i}<c_{3}(k) \max \left(1,\left|a_{1}\right|, \ldots, \mid a_{s}\right)^{c_{i}(k)}
$$

where $c_{2}$ and $c_{4}$ are explicit. Under suitable conditions and if $s$ is very large, the estimation can be considerably improved. (See, B. J. Birch [2] and W. M. Schmidt [11], [12].) In particular, Schmidt proved that if $s \geqslant c_{5}(k, \varepsilon)$, the equation

$$
a_{1} x_{1}^{k}+\ldots+a_{s} x_{s}^{k}=b_{1} y_{1}^{k}+\ldots+b_{s} y_{s}^{k}
$$

with positive rational integer coefficients has a nontrivial solution in nonnegative rational integers $x_{1}, \ldots, x_{s}, y_{1}, \ldots, y_{s}$ such that

$$
\max _{i, j}\left(x_{i}, y_{j}\right) \leqslant \max _{i, j}\left(a_{i}, b_{j}\right)^{1 / k+\varepsilon} .
$$

We use hereafter $\varepsilon, \varepsilon_{1}, \ldots$ to denote arbitrary preassigned positive numbers $<1$. The number $1 / k$ in (2) is best possible. Although the circle method is still used in the proof of (2), the treatment of the minor arcs is completely distinct from that in Waring's problem.

\footnotetext{
* Supported by the Institute for Advanced Study, Princeton, N. J. 08540.
} 